ARTICLE

DOI: $10.1038 / \mathrm{s} 41467-018-04097-9$

\title{
A biochemical framework for anaerobic oxidation of methane driven by Fe(III)-dependent respiration
}

\author{
Zhen Yan', Prachi Joshi (iD ${ }^{2}$, Christopher A. Gorski² \& James G. Ferry ${ }^{1}$
}

Consumption of methane by aerobic and anaerobic microbes governs the atmospheric level of this powerful greenhouse gas. Whereas a biochemical understanding of aerobic methanotrophy is well developed, a mechanistic understanding of anaerobic methanotrophy has been prevented by the unavailability of pure cultures. Here we report a biochemical investigation of Methanosarcina acetivorans, a methane-producing species capable of anaerobic methanotrophic growth dependent on reduction of Fe(III). Our findings support a pathway anchored by $\mathrm{Fe}(\mathrm{III})$-dependent mechanisms for energy conservation driving endergonic reactions that are key to methanotrophic growth. The pathway is remarkably similar to pathways hypothesized for uncultured anaerobic methanotrophic archaea. The results contribute to an improved understanding of the methane cycle that is paramount to understanding human interventions influencing Earth's climate. Finally, the pathway enables advanced development and optimization of biotechnologies converting methane to value-added products through metabolic engineering of $M$. acetivorans.

\footnotetext{
${ }^{1}$ Department of Biochemistry and Molecular Biology, Penn State, University Park, PA 16801, USA. ${ }^{2}$ Department of Civil and Environmental Engineering, Penn State, University Park, PA 16801, USA. Correspondence and requests for materials should be addressed to J.G.F. (email: jgf3@psu.edu)
} 
$\mathrm{T}$ he production and consumption of methane is a component of the global carbon cycle. An end product of the anaerobic decomposition of biomass, nearly one billion tons of methane is produced each year in diverse anaerobic habitats of the Earth's terrestrial biosphere ${ }^{1}$. Methane is also produced in anaerobic marine sediments and released from vast reservoirs of gas hydrates $\left(5.0 \times 10^{5}-1.0 \times 10^{7} \mathrm{Tg}\right)^{2}$. Methane is an important greenhouse gas nearly 20 -fold more potent than $\mathrm{CO}_{2}{ }^{3}$. Fortunately, release to the atmosphere is mitigated by the aerobic and anaerobic oxidation of methane or assimilation into cell biomass by microbes that ultimately control the Earth's climate ${ }^{4}$.

The anaerobic oxidation of methane (AOM) requires reduction of electron acceptors ( $\mathrm{Fe}(\mathrm{III}), \mathrm{Mn}(\mathrm{IV})$, nitrate or sulfate) to be thermodynamically favorable ${ }^{5,6}$. Until recently, it was thought that $\mathrm{AOM}$ in marine sediments required a symbiosis of anaerobic methanotrophic archaea (ANME) and sulfate-reducing species for which the latter utilizes reductant produced by the former to make the overall reaction thermodynamically favorable. However, the artificial electron acceptor AQDS (9,10-anthraquinone-2,6disulfonate), a surrogate for humic substances in the environment, decouples methane oxidation from sulfate reduction in marine sediments presenting the possibility of independent respiratory AOM and growth of usually syntrophic ANME- $2^{7,8}$. Indeed, respiratory AOM is in accordance with the occurrence of solitary ANME in microbial mats and sediments from diverse anaerobic environments ${ }^{9}$. Furthermore, incubations of environmental samples with $\mathrm{Fe}(\mathrm{III})$-citrate yielded biosynthetic activity exclusive to uncultured ANME-2c and other ANME-2 cells? These results inspired the hypotheses that symbiotic associations of ANME and sulfate-reducing species evolved from methanogenic species that first acquired the ability to conserve energy by oxidizing methane and reducing metal oxides ${ }^{7}$. $\mathrm{Fe}(\mathrm{III})$-dependent AOM reported for a culture enriched in 'Candidatus Methanoperedens nitroreducens' presents the possibility of $\mathrm{Fe}(\mathrm{III})$ dependent respiratory growth although not yet documented ${ }^{10}$. AOM dependent on reduction of $\mathrm{Fe}(\mathrm{III})$ is of particular biogeochemical interest. Indeed, it is proposed that if only a small percentage of current global $\mathrm{Mn}$ (IV) and $\mathrm{Fe}(\mathrm{III})$ influx is used for $\mathrm{AOM}$, it has the potential to consume a large amount of methane ${ }^{11}$. It is also proposed that $\mathrm{Fe}(\mathrm{III})$-dependent AOM was largely responsible for oxidizing the entirety of methane produced on early Earth prior to the advent of oxygen ${ }^{11}$.

The prospect that ANME are capable of independent AOM and growth by $\mathrm{Fe}(\mathrm{III})$ respiration profoundly changes current views of $\mathrm{AOM}$ and iron cycling in Nature, prompting further investigation. It is conjectured that ANME grow by reverse methanogenesis based on environmental metagenomic and metatranscriptomic analyses of sediments $s^{5,6}$. However, reversal and growth requires mechanisms for energy conservation and overcoming endergonic reactions yet to be investigated biochemically $^{5,6}$. Clearly, biochemical approaches with pure cultures are necessary to obtain a rigorous understanding of AOM. Although discovered nearly four decades ago, the unavailability of pure cultures has prevented biochemical investigations of AOM. However, a culture enriched in 'Candidatus Methanoperedens nitroreducens' is capable of AOM dependent on reduction of nitrate-, nitrite- or $\mathrm{Fe}(\mathrm{III})^{10,12}$. Furthermore, Methanosarcina acetivorans strain $\mathrm{C} 2 \mathrm{~A}$ is capable of trace methane oxidation (TMO) defined as reverse methanogenesis during net methane production from growth substrates in the absence of external electron acceptors ${ }^{6,13,14}$. TMO contrasts with AOM which is independent of methanogenesis and requires electron acceptors with or without a syntrophic partner. More recently, Fe(III)dependent AOM was documented for a strain of M. acetivorans engineered to produce methyl-coenzyme $M$ methyl reductase (Mcr) derived from ANME-1 sediments ${ }^{15}$. Thus, M. acetivorans has emerged as a model for advancing a biochemical understanding of AOM. M. acetivorans is also recognized as instrumental in development of biomanufacturing processes converting methane into value-added products ${ }^{16-18}$. Here we report a biochemical investigation of wild-type $M$. acetivorans that supports an AOM pathway anchored by $\mathrm{Fe}(\mathrm{III})$-dependent respiration generating ion gradients that supply the energy to drive endergonic reactions essential for AOM and growth. The results provide a deeper mechanistic understanding of $\mathrm{AOM}$ and iron cycling in Nature, and a guide for optimization of methane-based biotechnologies.

\section{Results}

Biochemistry of M. acetivorans resembles proposals for AOM. Mechanisms for energy conservation and driving endergonic reactions are key to reversing methanogenesis and methanotrophic growth (AOM). Based on environmental metagenomic and transcriptomic analyses, AOM pathways are proposed for ANME that include membrane-bound components involved in energy conservation (Rnf and Fpo complexes), electron transport (CoMS-SCoB heterodisulfide reductase (HdrDE); multi-heme $c$-type cytochromes (MHC); and methanophenazine (MP)) and methyl transfer (methyl-tetrahydrosarcinapterin:coenzyme M methyltransferase $(\mathrm{Mtr}))^{5,6,19-21}$. These components are essential to biochemically characterized methanogenic pathways in M. acetivorans strain C2A. Thus, the components were investigated in strain $\mathrm{C} 2 \mathrm{~A}$ to provide a biochemical understanding of roles in AOM of ANME.

Driving endergonic methane oxidation to the methyl level. The finding that both acetate and $\mathrm{CO}_{2}$ are produced during TMO and $\mathrm{AOM}$ by $M$. acetivorans indicates a dependence on reversal of acetoclastic and $\mathrm{CO}_{2}$-reducing methanogenic pathways, albeit with essential modifications ${ }^{13-15}$. The first two reactions required for reversal are methane oxidation yielding methyl-coenzyme $\mathrm{M}\left(\mathrm{CH}_{3}\right.$ $\mathrm{SCoM}$ ) and transfer of the methyl group to tetrahydrosarcinapterin $\left(\mathrm{H}_{4} \mathrm{SPT}\right)$. Both reactions are endergonic (Supplementary Table 1). The coenzyme M (HSCoM) and coenzyme B (HSCoB) products are oxidized to the heterodisulfide (CoMS-SCoB) which is the electron acceptor for the methane oxidation reaction yielding $\mathrm{CH}_{3}$ SCoM and HSCoB. The exergonic oxidation of HSCoM and HSCoB coupled to $\mathrm{Fe}(\mathrm{III})$ reduction is a potential mechanism driving the endergonic methane oxidation and methyl transfer reactions (Supplementary Table 1). This was tested by monitoring HSCoM and HSCoB oxidation by everted membrane vesicles prepared from acetate-grown $M$. acetivorans. The initial rate of free thiol consumption by vesicles loaded with S-layer-permeable $\mathrm{Fe}$ (III) citrate and AQDS ( $46.2 \mathrm{nmol} / \mathrm{min} / \mathrm{mg}$ vesicle protein) is 2.5 and 12.1-fold greater than the rate by vesicles loaded with only AQDS $(18.5 \mathrm{nmol} / \mathrm{min} / \mathrm{mg}$ vesicle protein) or only $\mathrm{Fe}(\mathrm{III})$ citrate (3.8 $\mathrm{nmol} / \mathrm{min} / \mathrm{mg}$ vesicle protein) (Fig. 1). As HdrDE is the only membrane-bound heterodisulfide reductase ${ }^{22-24}$, the results show that $\mathrm{HdrDE}$ oxidizes HSCoM/HSCoB with transfer of electrons to $\mathrm{Fe}(\mathrm{III})$ mediated by AQDS with the potential for product removal driving the endergonic oxidation of methane and transfer of the methyl group to $\mathrm{H}_{4} \mathrm{SPT}$.

Generation of a sodium gradient. Two membrane-bound complexes generate a $\mathrm{Na}^{+}$gradient (high outside) in the acetoclastic pathway of $M$. acetivorans ${ }^{22}$. The Rnf complex catalyzes the vectorial translocation of $\mathrm{Na}^{+}$generating a gradient (high outside) when oxidizing ferredoxin $\left(\mathrm{Fdx}_{\mathrm{R}}\right)$ with transfer of electrons to an electron transport chain culminating in reduction of CoMS-SCoB ${ }^{25-27}$. A Na+ gradient is also generated by the exergonic transfer of the methyl group from $\mathrm{CH}_{3}-\mathrm{H}_{4} \mathrm{SPT}$ to HSCoM by the methyltransferase (Mtr) ${ }^{22}$. A Rnf homolog plays a 


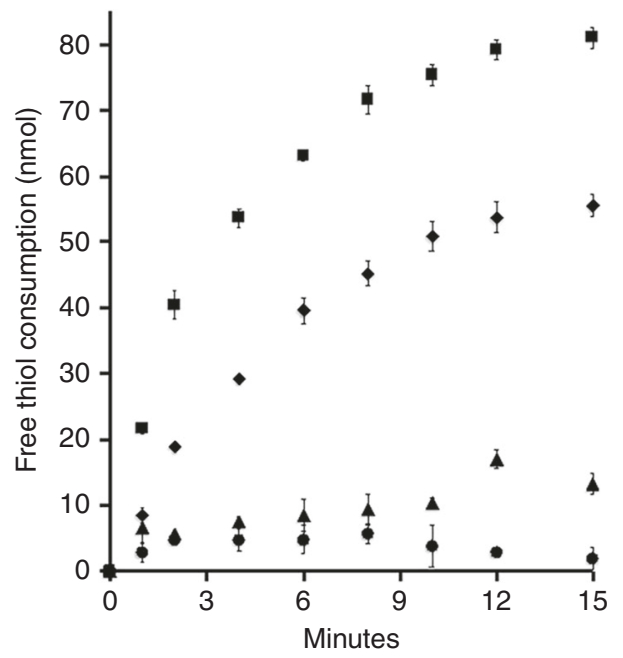

Fig. 1 Reverse heterodisulfide reductase activity of everted membrane vesicles dependent on $\mathrm{Fe}(\mathrm{III})$ as electron acceptor. The standard reaction mixture $(1.0 \mathrm{ml})$ contained $1.0 \mathrm{mM}$ each of $\mathrm{HSCoM}$ (coenzyme $\mathrm{M}$ ) and $\mathrm{HSCoB}$ (coenzyme B) in $50 \mathrm{mM}$ Tris ( $\mathrm{pH} \mathrm{8.0)} \mathrm{and} 1.0$ atmosphere Ar. The reaction was started by adding everted membrane vesicles $(0.5 \mathrm{mg}$ protein) prepared from acetate-grown cells that were empty (filled circles), loaded with Fe(III) (filled triangles), loaded with AQDS (9,10-anthraquinone -2,6-disulfonate) (filled diamonds) or loaded with Fe(III) plus AQDS (filled squares). Vesicles loaded with Fe(III) plus AQDS produced $88.4 \pm 5.4 \mathrm{nmol}$ $\mathrm{Fe}(\mathrm{II})$ at $15 \mathrm{~min}$ consistent with the expected stoichiometry. No free thiol consumption was detected (limit $=0.5 \mathrm{nmol}$ ) if only $\mathrm{HSCOM}$ or only $\mathrm{HSCoB}$ replaced HSCoM plus HSCoB. Data points are the mean of three biological replicates for which the standard deviation is shown by error bars

role in generating a $\mathrm{Na}^{+}$gradient in the AOM pathway proposed for an uncultured ANME-2a organism based on the metagenome and transcriptome ${ }^{6,19,21}$. This known biochemistry raised the question of whether oxidation of $\mathrm{Fdx}_{\mathrm{R}}$ by everted vesicles of $M$. acetivorans is coupled to reduction of $\mathrm{Fe}(\mathrm{III})$ that drives formation of a $\mathrm{Na}^{+}$gradient of sufficient magnitude to reverse the exergonic methyl transfer catalyzed by Mtr necessary for reversal of the acetoclastic and $\mathrm{CO}_{2}$-reduction methanogenic pathways.

$\mathrm{Fe}(\mathrm{III})$-loaded everted vesicles catalyze $\mathrm{Fdx}_{\mathrm{R}}$ : $\mathrm{Fe}(\mathrm{III})$ oxidoreductase (ORase) activity independent of AQDS and with rates similar to that of vesicles loaded with both AQDS and $\mathrm{Fe}(\mathrm{III})$, although sixfold greater than that of vesicles loaded with only AQDS (Supplementary Fig. 1). The rate of Fe(III)-dependent $\mathrm{Fdx}_{\mathrm{R}}$ oxidation is fourfold less with vesicles prepared from methanol-grown cells, a result consistent with a role for the Rnf complex that is downregulated in methanol- vs. acetate-grown $M$. acetivorans $^{25,26,28}$. Furthermore, Rnf is the only membranebound system capable of oxidizing $\mathrm{Fdx}_{\mathrm{R}}{ }^{23,25,27}$. The results establish that oxidation of $\mathrm{Fdx}_{\mathrm{R}}$ is coupled to the reduction of $\mathrm{Fe}$ (III) $\left(\Delta E^{o^{\prime}}=1.17 \mathrm{~V}\right)$ capable of generating a $\mathrm{Na}^{+}$gradient driving reversal of methyl transfer by Mtr.

A multi-heme c-type cytochrome (MHC), abundant in membranes of acetate-grown $M$. acetivorans, is reduced with $\mathrm{Fdx}_{\mathrm{R}}$ and re-oxidized by addition of $\mathrm{Fe}(\mathrm{III})$-citrate independent of AQDS (Supplementary Fig. 2), indicating a role for MHC in $\mathrm{Fdx}_{\mathrm{R}}: \mathrm{Fe}(\mathrm{III})$ ORase activity ${ }^{26}$. The failure of AQDS to enhance $\mathrm{Fdx}_{\mathrm{R}}: \mathrm{Fe}(\mathrm{III})$ ORase activity in everted vesicles (Supplementary Fig. 1) indicates direct transfer of electrons from a membranebound electron carrier to soluble $\mathrm{Fe}(\mathrm{III})$ citrate. The MHC is a strong candidate as it is the direct electron acceptor of the Rnf complex that oxidizes $\mathrm{Fdx}_{\mathrm{R}}{ }^{25,27}$. Whole cells reduce insoluble
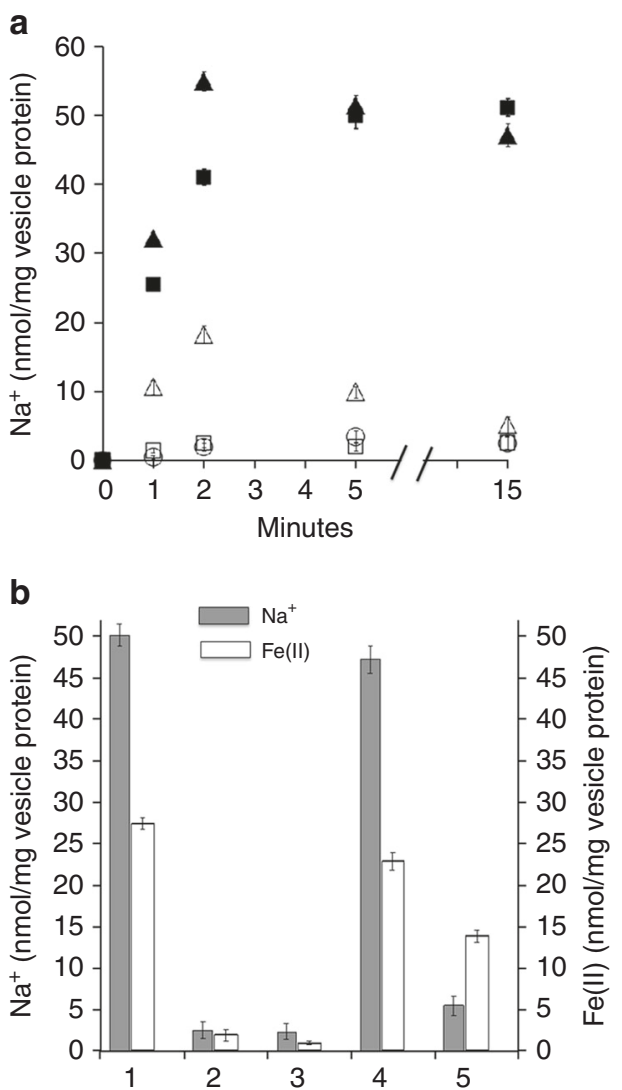

Fig. 2 Accumulation of ${ }^{22} \mathrm{Na}^{+}$in the lumen of everted membrane vesicles dependent on ferredoxin:Fe(III) oxidoreductase activity. The standard reaction mixture $(1.0 \mathrm{ml})$ contained Fe(III)-loaded vesicles ( $1.0 \mathrm{mg}$ protein) from acetate-grown cells, $5.0 \mu \mathrm{M} \mathrm{Fdx}$ (ferredoxin), $0.5 \mathrm{mM} \mathrm{NaCl}, 0.5 \mu \mathrm{Ci}$ / $\mathrm{ml}^{22} \mathrm{NaCl}$ (carrier free), $25 \mathrm{mM} \mathrm{MgSO}_{4}, 0.4 \mathrm{M}$ sucrose and $10 \mathrm{mM}$ dithiothreitol in $50 \mathrm{mM}$ Tris ( $\mathrm{pH}$ 8.0) and 1.0 atmosphere of $\mathrm{CO}$. Reactions were initiated by addition of $\mathrm{CO}$ dehydrogenase/acetyl-CoA synthase $(0.1$ $\mathrm{mg}$ ). a Time course of accumulation. Data points are the mean of three biological replicates for which the standard deviation is shown by error bars. Standard reaction mixture (filled squares), minus Fdx (empty squares), with empty vesicles (empty circles), plus $10 \mu \mathrm{M}$ carbonyl cyanide $m$-chlorophenyl hydrazine (CCCP) (filled triangles), plus $10 \mu \mathrm{M}$ ETH157 (empty triangles). No accumulation occurred if $\mathrm{N}_{2}$ replaced $\mathrm{CO}$. b Sodium and Fe(II) accumulation in the lumen of vesicles at the 15-min time point shown in $\mathbf{a}$. The standard reaction mixtures were modified as indicated for each bar: (1) standard, no modification, (2) minus Fdx, (3) empty vesicles, (4) plus $10 \mu \mathrm{M}$ CCCP, (5) plus $10 \mu \mathrm{M}$ ETH157. Bars are the mean of three biological replicates for which the standard deviation is shown by error bars

ferrihydrite with $\mathrm{CO}$ as the electron donor for which the initial rate is stimulated fourfold in the presence of AQDS, albeit fourfold less than with Fe(III) citrate (Supplementary Fig. 3). This result indicates that AQDS is essential to mediate electron transfer to insoluble $\mathrm{Fe}(\mathrm{III})$ oxides.

The $\mathrm{Fdx}_{\mathrm{R}}: \mathrm{Fe}(\mathrm{III})$ ORase activity of $\mathrm{Fe}(\mathrm{III})$ citrate-loaded everted vesicles is stimulated fourfold by the addition of $\mathrm{NaCl}$ to the reaction mixture that is concentration dependent with a $K_{\mathrm{m}}$ of $\sim 1.0 \mathrm{mM}$ (Supplementary Fig. 1). The stimulation is confirmed with vesicles loaded with both AQDS and Fe(III) citrate. The results are consistent with $\mathrm{Na}^{+}$translocation into the lumen driven by electron transport. The apparent translocation of $\mathrm{Na}^{+}$ was further investigated with ${ }^{22} \mathrm{Na}^{+}$that rapidly accumulates in everted vesicles $(25 \mathrm{nmol} / \mathrm{mg}$ protein/min) dependent on $\mathrm{Fe}(\mathrm{III})$ 
and $\mathrm{Fdx}_{\mathrm{R}}$ (Fig. 2a). The $\mathrm{Na}^{+}$ionophore ETH157 abolishes accumulation. These results establish that membranes translocate $\mathrm{Na}^{+}$coupled to electron transport from $\mathrm{Fdx}_{\mathrm{R}}$ to $\mathrm{Fe}(\mathrm{III})$. The protonophore CCCP (carbonyl cyanide $m$-chlorophenyl hydrazine) has no effect on the rate of $\mathrm{Na}^{+}$accumulation which precludes that $\mathrm{Na}^{+}$translocation is a secondary effect driven by a primary $\mathrm{H}^{+}$gradient combined with a Na${ }^{+} / \mathrm{H}^{+}$antiporter. Accumulation of $\mathrm{Fe}$ (II) in vesicles correlates with accumulation of ${ }^{22} \mathrm{Na}^{+}$for each experimental condition except as expected for addition of ETH157 (Fig. 2b). Vesicles loaded with Fe(III) completely oxidize limiting amounts of fully reduced $\mathrm{Fdx}_{\mathrm{R}}{ }^{2-}(2.5$ nmol) and accumulate a total of $4.7 \pm 0.4(n=3)$ nmol Fe(II) consistent with the expected stoichiometry. The ratio of ${ }^{22} \mathrm{Na}^{+} / \mathrm{Fe}$ (II) is $1.8 \pm 0.2$ indicating translocation of two $\mathrm{Na}^{+} /$electron transported. Generation of a $\mathrm{Na}^{+}$gradient by Rnf and the highly exergonic $\mathrm{Fe}(\mathrm{III})$-dependent oxidation of the reaction product HSCoM by HdrDE are potential mechanisms for driving the endergonic methyl transfer reaction by Mtr.

Driving endergonic methyl transfer. It was next asked if the $\mathrm{Na}$ + gradient generated by $\mathrm{Fdx}_{\mathrm{R}}: \mathrm{Fe}(\mathrm{III})$ ORase activity is of sufficient magnitude to drive the endergonic methyl transfer from $\mathrm{CH}_{3}$ SCoM to $\mathrm{H}_{4}$ SPT by Mtr. Tetrahydrofolate (THF), a functional homolog of $\mathrm{H}_{4} \mathrm{SPT}$, was used in the experiments ${ }^{29}$. Membranes catalyze methyl transfer in the thermodynamically favorable direction of $\mathrm{CH}_{3}$-SCoM synthesis with the methyl group donated by $\mathrm{CH}_{3}$-THF (Supplementary Fig. 4). The maximum initial rate of activity $(180.8 \pm 4.2 \mathrm{nmol} / \mathrm{min} / \mathrm{mg}$ protein) is dependent on carbon monoxide (CO) and adenosine triphosphate (ATP), a result characteristic of reductive activation for Mtr of

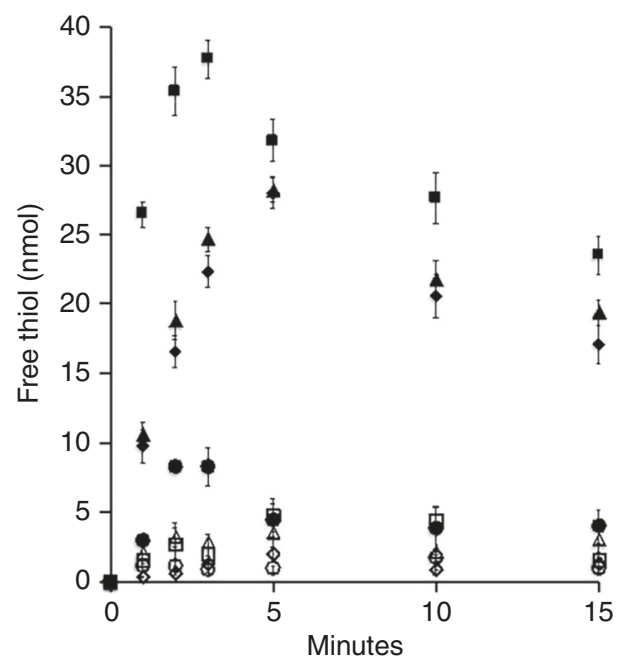

Fig. 3 Reverse methyltransferase activity in the direction of $\mathrm{CH}_{3}-$ tetrahydrofolate production driven by a sodium gradient dependent on reduced ferredoxin:Fe(III) oxidoreductase activity. The standard reaction mixture $(1.0 \mathrm{ml})$ contained Fe(III)-loaded vesicles (1.0 mg protein), $2.0 \mathrm{mM}$ $\mathrm{CH}_{3}$-SCoM (methyl-coenzyme M), $2.0 \mathrm{mM} \mathrm{THF}$ (tetrahydrofolate), $5.0 \mu \mathrm{M}$ Fdx (ferredoxin), $25 \mathrm{mM} \mathrm{MgSO}_{4}, 0.4 \mathrm{M}$ sucrose and $0.5 \mathrm{mM}$ residual $\mathrm{NaCl}$ in $20 \mathrm{mM}$ Tris ( $\mathrm{pH} \mathrm{7.0)}$ and 1.0 atmosphere of $\mathrm{CO}$. Reactions were initiated with $\mathrm{CO}$ dehydrogenase/acetyl-CoA synthase $(0.1 \mathrm{mg})$. Standard reaction mixture (filled diamonds), plus $1 \mathrm{mM}$ ATP (filled squares), plus $10 \mu \mathrm{M}$ carbonyl cyanide $m$-chlorophenyl hydrazine (CCCP) (filled triangles), plus $10 \mu \mathrm{M}$ ETH157 (filled circles), minus Fdx (empty squares), with empty vesicles (empty diamonds), minus $\mathrm{CH}_{3}$-SCoM (empty circles), minus THF (empty triangles). Data points are the mean of three biological replicates for which the standard deviation is shown by the error bars
Methanosarcina species ${ }^{29}$. Everted membrane vesicles catalyze Fdx- and $\mathrm{Fe}(\mathrm{III})$-dependent methyl transfer from $\mathrm{CH}_{3}-\mathrm{SCoM}$ to THF with an initial rate of $9.8 \pm 1.2 \mathrm{nmol} / \mathrm{min} / \mathrm{mg}$ protein that is abolished by the $\mathrm{Na}^{+}$ionophore ETH157 (Fig. 3). The presence of ATP stimulates the initial rate to $26.5 \pm 0.9 \mathrm{nmol} / \mathrm{min} / \mathrm{mg}$ protein consistent with results reported for $\mathrm{Mtr}^{29}$. The results demonstrate that the $\mathrm{Na}^{+}$gradient generated by $\mathrm{Fdx}_{\mathrm{R}}$ : $\mathrm{Fe}$ (III) ORase activity is of sufficient magnitude to drive the endergonic methyl transfer from $\mathrm{CH}_{3}-\mathrm{SCoM}$ to $\mathrm{H}_{4} \mathrm{SPT}$.

Conservation of energy. The mechanisms by which energy is conserved in ATP for growth of ANME is of fundamental importance in addition to mechanisms driving essential endergonic reactions. Substrate-level phosphorylation catalyzed by acetate kinase is a potential mechanism for ATP synthesis when considering acetate is a product of $\mathrm{AOM}$ by M. acetivorans ${ }^{15}$. Another possibility is ATP synthesis catalyzed by the ATP synthase which is dependent on both $\mathrm{Na}^{+}$and $\mathrm{H}^{+}$gradients ${ }^{30}$. This mechanism requires generation of a $\mathrm{H}^{+}$gradient to accompany the $\mathrm{Na}^{+}$gradient generated by $\mathrm{Fdx}_{\mathrm{R}}$ : $\mathrm{Fe}$ (III) ORase activity. The membrane-bound Fpo complex catalyzes a vectorial translocation of $\mathrm{H}^{+}$generating a gradient (high outside) by oxidizing coenzyme $\mathrm{F}_{420} \mathrm{H}_{2}$ with transfer of electrons to $\mathrm{HdrDE}\left(\mathrm{F}_{420} \mathrm{H}_{2}\right.$ :CoB-S-SCoM ORase activity) ${ }^{31}$. Based on metagenomic and transcriptomic analyses, Fpo homologs are proposed to play a role in generating $\mathrm{H}^{+}$gradients in AOM pathways based on metagenomic and metatranscriptomic analyses of ANME ${ }^{5,19-21,32}$ which prompted asking if the oxidation of $\mathrm{F}_{420} \mathrm{H}_{2}$ by the Fpo of $M$. acetivorans is coupled to reduction of $\mathrm{Fe}(\mathrm{III})\left(\mathrm{F}_{420} \mathrm{H}_{2}\right.$ : $\mathrm{Fe}$ (III) ORase activity) that also generates a $\mathrm{H}^{+}$gradient. Fpo activity is substantial in methanol-grown $M$. acetivorans ${ }^{28,33}$ from which $\mathrm{Fe}(\mathrm{III})$ citrateloaded everted membrane vesicles were prepared to evaluate $\mathrm{F}_{420} \mathrm{H}_{2}: \mathrm{Fe}$ (III) ORase activity. The vesicles catalyze $\mathrm{Fe}(\mathrm{III})$ dependent $\mathrm{F}_{420} \mathrm{H}_{2}$ oxidation (Supplementary Fig. 5) with a sevenfold stimulation of the initial rate upon addition of the protonophore CCCP, indicating electron transport coupled to $\mathrm{H}^{+}$ translocation. The rate is fourfold less with vesicles from acetategrown cells corresponding to fivefold less Fpo, a result supporting a role for Fpo in Fe(III)-dependent $\mathrm{F}_{420} \mathrm{H}_{2}$ oxidation. Vesicles accumulate a total of $11.2 \pm 1.1 \mathrm{nmol}(n=3) \mathrm{Fe}$ (II) in reaction mixtures containing $5.0 \mathrm{nmol} \mathrm{F}_{420} \mathrm{H}_{2}$ that is oxidized to completion (not shown), a result expected for the obligate twoelectron carrier ${ }^{34}$. Negligible $\mathrm{Fe}(\mathrm{II})$ is produced in the absence of $\mathrm{F}_{420} \mathrm{H}_{2}$ or $\mathrm{Fe}$ (III).

The finding that CCCP stimulates $\mathrm{F}_{420} \mathrm{H}_{2}$-dependent reduction of $\mathrm{Fe}$ (III) prompted experiments to determine if the highly exergonic electron transport $\left(\Delta E^{O^{\prime}}=1.15 \mathrm{~V}\right)$ is coupled to vectorial pumping of $\mathrm{H}^{+}$from the medium into the lumen of everted vesicles loaded with $\mathrm{Fe}$ (III) citrate (Fig. 4). Addition of $\mathrm{F}_{420} \mathrm{H}_{2}$ produces rapid alkalinization of the medium followed by gradual acidification towards stabilization of the $\mathrm{pH}$ (Fig. 4a). The ATPase inhibitor, $N, N^{\prime}$-dicyclohexylcarbodiimide (DCCD), has no effect on $\mathrm{H}^{+}$translocation, ruling out a role for ATP-driven translocation. Negligible alkalinization and re-acidification is observed with vesicles devoid of $\mathrm{Fe}(\mathrm{III})$ or when $\mathrm{F}_{420}$ replaces $\mathrm{F}_{420} \mathrm{H}_{2}$ (not shown). Increased concentrations of the protonophore CCCP (carbonyl cyanide $m$-chlorophenyl hydrazine) correlate with decreased $\mathrm{F}_{420} \mathrm{H}_{2}$-dependent alkalinization (Fig. 4b). The results demonstrate that the electron transport is coupled to translocation of $\mathrm{H}^{+}$from the medium into the lumen. Increased alkalinization correlates with increased amounts of $\mathrm{F}_{420} \mathrm{H}_{2}$ from which it is calculated that $2.1 \mathrm{H}^{+}$enters into the lumen per $\mathrm{F}_{420} \mathrm{H}_{2}$ oxidized (Fig. 4c). However, the results indicate translocation of $4 \mathrm{H}^{+}$when considering $2 \mathrm{H}^{+}$are produced outside the vesicle upon oxidation of $\mathrm{F}_{420} \mathrm{H}_{2}$. The result is 
a

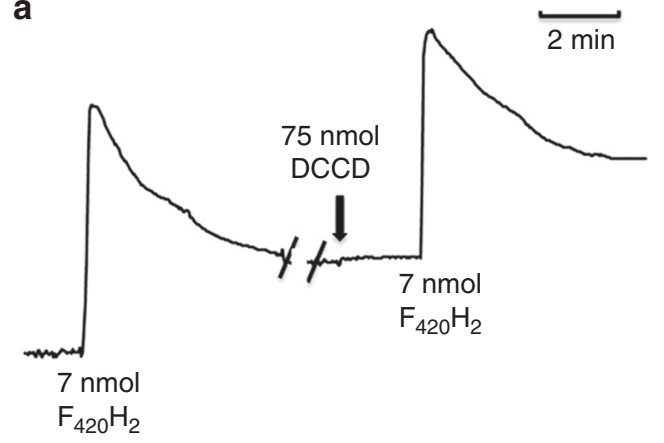

b

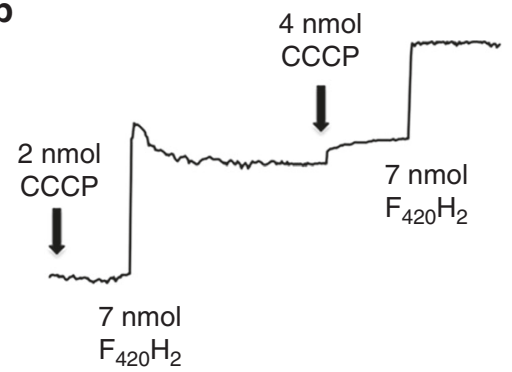

C

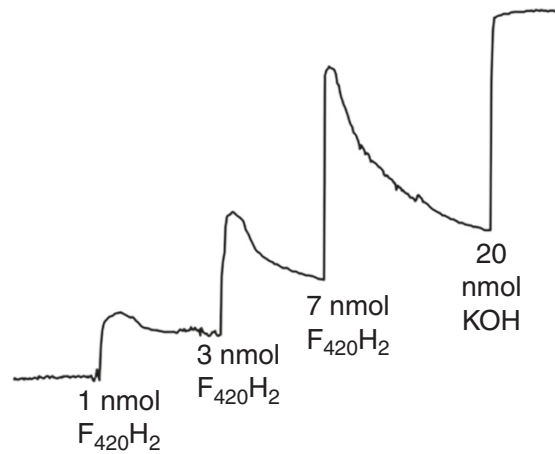

Fig. 4 Proton uptake in everted membrane vesicles dependent on coenzyme $\mathrm{F}_{420} \mathrm{H}_{2}: \mathrm{Fe}(\mathrm{III})$ oxidoreductase activity. The reaction mixtures $(2.0 \mathrm{ml})$ contained $40 \mathrm{mM} \mathrm{KSCN}, 0.4 \mathrm{M}$ sucrose, $10 \mathrm{mM}$ dithiothreitol and $\mathrm{Fe}(\mathrm{III})$-loaded vesicles ( $0.5 \mathrm{mg}$ protein) from methanol-grown cells in 2 $\mathrm{mM}$ potassium phosphate buffer $(\mathrm{pH} 7.0)$ and the indicated final concentration of reduced coenzyme $\mathrm{F}_{420}\left(\mathrm{~F}_{420} \mathrm{H} \mathrm{H}_{2}\right)$. Additional reagents were added as indicated with final concentrations shown. a Effect of dicyclohexylcarbodiimide (DCCD). $\mathbf{b}$ Effect of carbonyl cyanide $m$ chlorophenyl hydrazine (CCCP). c Effect of increasing concentrations of $\mathrm{F}_{420} \mathrm{H}_{2}$. The difference between the starting baseline and the final baseline represents alkalinization of the medium dependent on additions

consistent with the stoichiometry predicted for the $\mathrm{F}_{420} \mathrm{H}_{2}$ :CoMSSCoB ORase activity of Methanosarcina species ${ }^{35}$. Although all the electron transfer components have yet to be determined, the results establish that $\mathrm{F}_{420} \mathrm{H}_{2}: \mathrm{Fe}$ (III) ORase activity is coupled to generation of a $\mathrm{H}^{+}$gradient with the potential to assist in ATP synthesis by the $\mathrm{Na}^{+}$- and $\mathrm{H}^{+}$-dependent ATP synthase.

Proposed pathway of $\mathrm{Fe}(\mathrm{III})$-dependent $\mathrm{AOM}$ and methanotrophic growth. Acetate and $\mathrm{CO}_{2}$ are products of $\mathrm{Fe}(\mathrm{III})$ dependent AOM by $M$. acetivorans indicating reversal of both acetoclastic and $\mathrm{CO}_{2}$-reducing methanogenic pathways ${ }^{15}$. Figure 5 shows a pathway proposed for soluble Fe(III)-dependent AOM by $M$. acetivorans based on results presented here and characterized components of the well-established acetoclastic and $\mathrm{CO}_{2}$-reducing methanogenic pathways of $M$. acetivorans ${ }^{23,25-28,33,36,37}$. As the outer S-layer of $M$. acetivorans contains pores of sufficient size to permit passage of $\mathrm{Fe}(\mathrm{III})$-citrate, results obtained with everted vesicles can be extrapolated to whole cells ${ }^{38}$.

In the proposed pathway (Fig. 5), methane is assumed oxidized by the methyl-coenzyme $M$ methyl reductase (Mcr) (Rxn. 1) based on TMO and AOM by M. acetivorans that requires Mcr activity ${ }^{13-15}$. The methyl group of $\mathrm{CH}_{3}-\mathrm{SCoM}$ is then transferred to $\mathrm{H}_{4} \mathrm{SPT}$ by the membrane-bound methyltransferase (Mtr) (Rxn. 2). The results presented show the endergonic reaction is driven by the $\mathrm{Na}^{+}$gradient generated with $\mathrm{Fdx}_{\mathrm{R}}$ : $\mathrm{Fe}(\mathrm{III})$ ORase activity catalyzed by the Rnf complex (Rxn. 3$)^{23,27}$. The results also indicate that $\mathrm{MHC}$ is the direct electron donor to $\mathrm{Fe}(\mathrm{III})$, analogous to reduction of soluble and insoluble forms of $\mathrm{Fe}$ (III) by the MHC of $\mathrm{Fe}(\mathrm{III})$ reducing microbes such as Shewanella oneidensis ${ }^{39,40}$.

The HSCoM and HSCoB products of reactions 1 and 2 are oxidized to regenerate CoMS-SCoB essential for Mcr-catalyzed methane oxidation (Rxn. 1). The results presented indicate the oxidation is catalyzed by the membrane-bound HdrDE (Rxn. 4) dependent on AQDS-mediated reduction of $\mathrm{Fe}(\mathrm{III})$. The endergonic oxidation of methane (Rxn. 1) is driven by exergonic reaction 4 coupled with reaction 2 driven by exergonic reaction 3 (Supplementary Table 1). The path of AQDS-dependent electron transport from HdrDE to $\mathrm{Fe}(\mathrm{III})$ is unknown. MP is a quinonelike, membrane-intrinsic, electron carrier that donates to cytochrome $b$ in all pathways of methanogenesis by $M$. acetivorans $^{23,27}$. Although reduction of MP by cytochrome $b$ is expected, $\mathrm{MPH}_{2}$ is also expected to directly reduce $\mathrm{Fe}(\mathrm{III})$ analogous to abiotic reduction of AQDS ${ }^{4,42}$ obviating a requirement for AQDS in contrast to that observed. Thus, a more plausible pathway involves a role for AQDS accepting electrons from cytochrome $b$ of the membrane-intrinsic HdrE and AQDS abiotically reducing Fe(III) (Fig. 5).

It is proposed that the methyl group of $\mathrm{CH}_{3}-\mathrm{H}_{4} \mathrm{SPT}$ enters branched pathways for production of acetate (Rxns. 5-6) and oxidation to $\mathrm{CO}_{2}$ (Rxns. 7-8). Transfer of electrons to $\mathrm{Fe}(\mathrm{III})$ in the $\mathrm{CO}_{2}$ oxidation branch is essential for the overall pathway to be thermodynamically favorable (Supplementary Table 1). The acetate branch is included based on previous findings that acetate is a product of $\mathrm{Fe}$ (III)-dependent AOM by M. acetivorans ${ }^{15}$. The exergonic reactions leading to acetate are experimentally validated for the pathway of CO-dependent growth by M. acetivorans ${ }^{33}$. Reactions proposed for oxidation of the methyl group of $\mathrm{CH}_{3}$ $\mathrm{H}_{4}$ SPT to $\mathrm{CO}_{2}$ (Rxns. 7 and 8) are components of the $\mathrm{CO}_{2^{-}}$ reduction pathway of $M$. acetivorans ${ }^{33}$. The pathway (Fig. 5) proposes that the $\mathrm{F}_{420} \mathrm{H}_{2}$ produced is re-oxidized with transfer of electrons to $\mathrm{Fe}$ (III) by two possible mechanisms. The first is by exergonic $\mathrm{F}_{420} \mathrm{H}_{2}: \mathrm{Fe}$ (III) ORase activity coupled to the generation of $\mathrm{a} \mathrm{H}^{+}$gradient ( $\mathrm{Rxn} .9$ ) involving Fpo that is also essential to the $\mathrm{CO}_{2}$-reduction pathway of $M$. acetivorans ${ }^{33}$. Although the path of electron transfer from $\mathrm{F}_{420} \mathrm{H}_{2}$ to $\mathrm{Fe}$ (III) is unknown, failure of AQDS to stimulate $\mathrm{F}_{420} \mathrm{H}_{2}: \mathrm{Fe}$ (III) ORase activity indicates electron transfer from Fpo to Fe(III) is independent of AQDS and HdrDE in contrast to electron transport from $\mathrm{HdrDE}$ to $\mathrm{Fe}$ (III). A role for $\mathrm{MP}$ is possible as it is the electron acceptor for $\mathrm{Fpo}^{23,35}$. It is proposed that the $\mathrm{MPH}_{2}$ generated is oxidized by $\mathrm{Fe}$ (III) contributing to the $\mathrm{H}^{+}$gradient (Fig. 5, Rxn. 9). Alternatively, $\mathrm{F}_{420} \mathrm{H}_{2}$ may be oxidized by the recently characterized electron bifurcating HdrA2B2C2 of $M$. acetivorans (Rxn. 10) with transfer of electrons to $\mathrm{Fdx}_{\mathrm{O}}$ (Rxn. 11) and CoMS-SCoB (Rxn. 12) ${ }^{43}$. Oxidation of HSCoM and HSCoB (Rxn. 13) is postulated to be accomplished by HdrDE with exergonic transfer of electrons to Fe (III) dependent on AQDS analogous to reaction 4. 


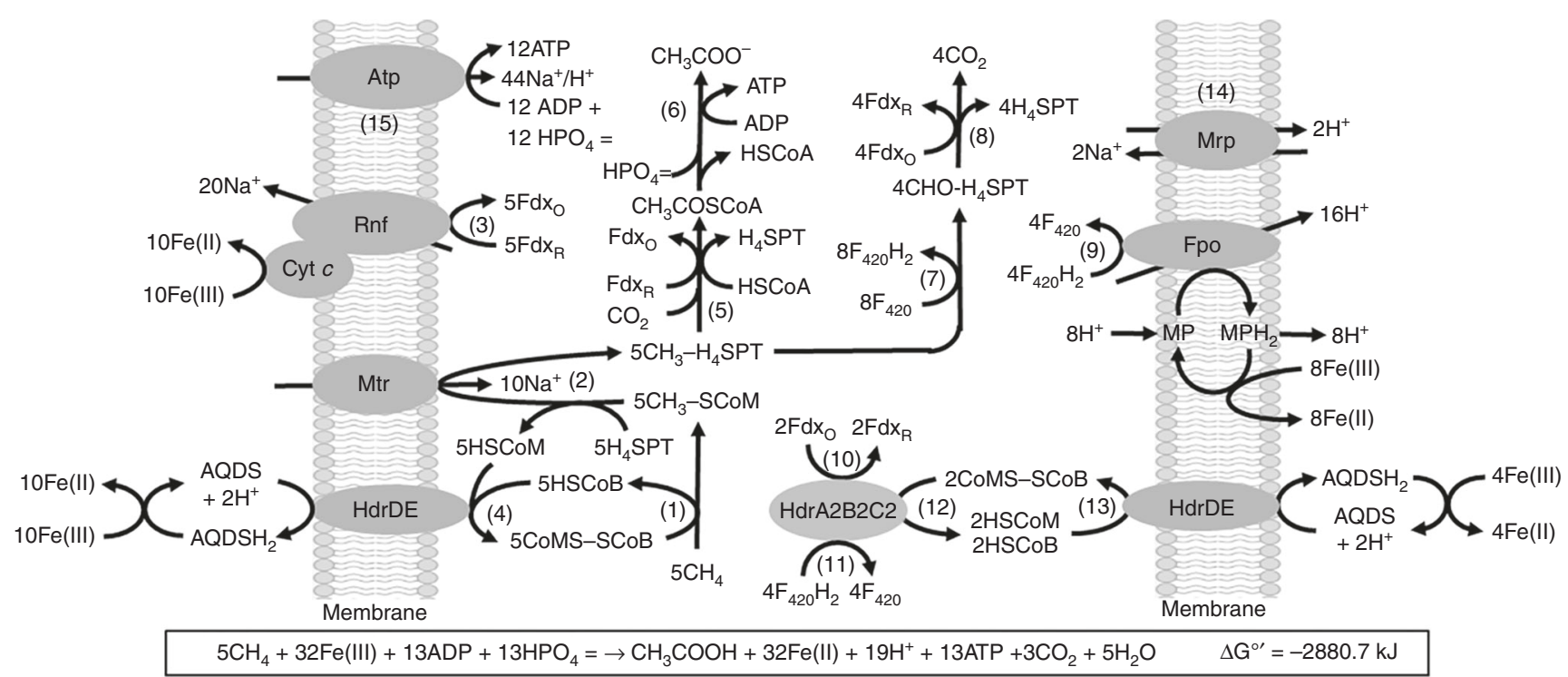

Fig. 5 Pathway proposed for Fe(III)-dependent methane oxidation and conservation of energy by M. acetivorans. Enzymes not discussed in the text: CO dehydrogenase/acetyl-CoA synthase (Rxn. 5); acetate kinase and phosphotransacetylase (Rxn. 6); coenzyme $F_{420}\left(F_{420}\right)$-dependent methylenetetrahydrosarcinapterin $\left(\mathrm{H}_{4} \mathrm{SPT}\right)$ reductase, $\mathrm{F}_{420}$-dependent methylene- $\mathrm{H}_{4} \mathrm{SPT}$ dehydrogenase, methenyl- $\mathrm{H}_{4} \mathrm{SPT}$ cyclohydrolase, and formylmethanofuran: $\mathrm{H}_{4} \mathrm{SPT}$ formyltransferase (Rxn. 7); formylmethanofuran dehydrogenase (Rxn. 8). MP, methanophenazine

Finally, it is proposed that the ratio of acetate/ $\mathrm{CO}_{2}$ produced is flexible and governs the ratio of $\mathrm{Na}^{+} / \mathrm{H}^{+}$gradients requiring the multi-subunit $\mathrm{Na}^{+} / \mathrm{H}^{+}$antiporter $\mathrm{Mrp}$ (Rxn. 14) to adjust the $\mathrm{Na}$ $+/ \mathrm{H}^{+}$ratio optimal for the ATP synthase shown previously to be dependent on both $\mathrm{Na}^{+}$and $\mathrm{H}^{+}(\text {Rxn. 15) })^{30,44}$.

\section{Discussion}

The proposed biochemical-based pathway elevates AOM by $M$. acetivorans to a fundamental mechanistic level of understanding. The pathway is essentially a reversal of the biochemically characterized acetoclastic and $\mathrm{CO}_{2}$-reduction methanogenic pathways, albeit dependent on reduction of $\mathrm{Fe}(\mathrm{III})$. The results reveal the role $\mathrm{Fe}(\mathrm{III})$ plays in energy conservation and driving endergonic reactions that support methanotrophic growth ${ }^{15}$. Abundant methane is targeted for development of recently advocated biomanufacturing processes converting it to liquid biofuels and value-added products ${ }^{16}$. Thus, the pathway provides a guide for optimization of existing, and development of advanced, biotechnologies facilitated by the timely announcement of a cas9-mediated genome editing system for facile metabolic engineering of $M$. acetivorans ${ }^{16-18,45-47}$.

The proposed $M$. acetivorans pathway is remarkably similar to the pathway proposed for ANME-2a based on the single-aggregate genome derived from integrated environmental metagenomic and transcriptomic analyses of a marine environment ${ }^{21}$. The $M$. acetivorans and ANME-2a pathways both include Rnf, Fpo, MHC, $\mathrm{HdrDE}$ and $\mathrm{CO}$ dehydrogenase/acetyl-CoA synthase for which the homologs show robust amino acid sequence identities (Supplementary Table 2). The results presented, showing generation of $\mathrm{Na}$ ${ }^{+}$and $\mathrm{H}^{+}$ion gradients by Rnf and Fpo of M. acetivorans, validate the same roles for Rnf and Fpo in pathways proposed for uncultured ANME $^{20,21,32}$. The pathways for $M$. acetivorans, ANME-1, ANME-2a and ANME-2d include acetate as a product ${ }^{12,21,32}$. Furthermore, analysis of methane seep sediments show robust ${ }^{13} \mathrm{C}$ depletion in acetate with $\delta^{13} \mathrm{C}$ values near $-90 \%{ }^{48}$. These results suggest that the proposed AOM pathway of M. acetivorans is largely representative of ANME, further strengthened by data showing ANME-1, ANME-2d and ANME-2a are phylogenetically related to Methanosarcina species ${ }^{10,20,21,49}$. The one exception is Mcr of wildtype M. acetivorans which could explain why only the engineered strain containing Mcr from ANME-1 is capable of Fe(III)-dependent $\mathrm{AOM}^{15}$. No other genetic alterations were imposed on the engineered strain ensuring that mechanisms for $\mathrm{Fe}(\mathrm{III})$-dependent energy conservation and driving endothermic reactions are identical to those investigated in the wild type. Although TMO by wild-type $M$. acetivorans indicates that Mcr catalyzes the reverse reaction analogous to that of Methanothermobacter marburgensis ${ }^{50}$, bias in the direction of methane formation may be a kinetic block to initiating $\mathrm{AOM}$ as opposed to a more favorable rate of methane oxidation for Mcr of ANME.

The similarity in pathways for M. acetivorans and proposed for ANME-2a strengthen the prospect of $\mathrm{Fe}(\mathrm{III})$-dependent AOM and respiratory growth by uncultured ANME. Importantly, the roles shown for Rnf, MHC, Fpo and HdrDE in electron transfer to $\mathrm{Fe}(\mathrm{III})$ by M. acetivorans are also hypothesized for ANME-2 in transfer of electrons to metal oxides or the syntrophic partner ${ }^{19}$. The MHCs of ANME- 2 are proposed to extend beyond the Slayer to facilitate the electron transfer. The results presented here indicate that soluble $\mathrm{Fe}(\mathrm{III})$ citrate is reduced by the MHC of $M$. acetivorans. It is conceivable that the MHC also plays a role in reducing insoluble forms of $\mathrm{Fe}(\mathrm{III})$. If so, either multiple MHCs extend to the outer S-layer of M. acetivorans or diffusible electron carriers such as low-molecular-mass humic acids or secreted flavins mediate electron transfer between the MHC and the Slayer ${ }^{7,51}$. The latter is consistent with the finding that AQDS, a surrogate for humics, stimulated ferrihydrite reduction in whole cells of M. acetivorans. Extension of MHCs to the outer S-layer is consistent with MHCs from ANME-2a and ANME-2d reported to be fused with a putative S-layer domain homologous to the S-layer protein of M. acetivorans ${ }^{19}$.

The pathway proposed for $M$. acetivorans is also consistent with the hypothesis that symbiotic associations of ANME and sulfate-reducing species evolved from methanogenic species that 
first acquired the ability to conserve energy by oxidizing methane coupled to reduction of metal oxides ${ }^{7}$. However, it is also possible that the ancestor common to M. acetivorans and ANME first performed AOM with a sulfate reducer and then switched to reduction of metal oxides. Unlike methanogenesis pathways, the proposed AOM pathway of $M$. acetivorans features multiple roles for heterodisulfide reductases (Fig. 5). Two types of heterodisulfide reductases are essential to all methanogenic pathways, the cytoplasmic HdrABC and the membrane-associated $\mathrm{HdrDE}^{23,24}$. The results presented here support a role for HdrDE coupled to AQDS-mediated reduction of Fe(III) that drives the endergonic methane oxidation and methyl transfer reactions essential to reversal. A role is proposed for the electron bifurcating $\mathrm{HdrA} 2 \mathrm{~B} 2 \mathrm{C} 2$ in energy conservation when generation of $\mathrm{Na}^{+}$and $\mathrm{H}^{+}$gradients by Rnf and Fpo (Fig. 5, Rxns. 3 and 9) are limited by the availability of $\mathrm{Fe}(\mathrm{III})$. The $\mathrm{HdrA} 2 \mathrm{~B} 2 \mathrm{C} 2$ plays this role by diverting electron flow from $\mathrm{F}_{420} \mathrm{H}_{2}$ to $\mathrm{Fdx}_{\mathrm{O}}$ promoting acetate production and ATP synthesis by substrate-level phosphorylation (Fig. 5, Rxns. 5 and 6). Notably, the genome of the ANME 'Candidatus Methanoperedens nitroreducens' encodes a homolog of HdrA2 that is the flavin-containing electronbifurcating subunit of $\mathrm{HdrA} 2 \mathrm{~B} 2 \mathrm{C} 2^{43,52}$.

A role for $\mathrm{H}_{2}$ in electron transport and energy conservation is unlikely for $\mathrm{Fe}(\mathrm{III})$-dependent AOM. M. acetivorans is incapable of metabolizing $\mathrm{H}_{2}$ which contrasts with other species of Methanosarcina for which ATP synthesis is dependent on the production and consumption of $\mathrm{H}_{2}$ to generate $\mathrm{H}^{+}$gradients ${ }^{23}$. The thermodynamically unfavorable production of $\mathrm{H}_{2}$ as an electron transfer agent could impose a barrier to reversing methanogenesis. Metagenomic analyses of the ANME-2a clade, phylogenetically related to $M$. acetivorans, are devoid of hydrogenase genes ${ }^{21}$. Thus, the ANME-2a clade is unlikely to employ $\mathrm{H}_{2}$ for transferring electrons to syntrophic partners. It is more likely that electrons are transferred via DIET (direct interspecies electron transfer) as previously hypothesized for uncultured species of the ANME- 2 clade $^{19}$. Pathways proposed for ANME include acetate as a product, although disputed as a diffusible electron carrier in syntrophic $\mathrm{AOM}^{12,19,21,32,53}$. If not involved in syntrophic AOM, acetate may be an essential carbon source for the syntrophic partner ${ }^{21}$. A portion of acetyl-CoA in the pathway of acetate production (Fig. 5) may also enter biosynthetic pathways essential for AOM by ANME and M. acetivorans ${ }^{54}$.

In addition to AOM, M. acetivorans is capable of TMO producing $\mathrm{CO}_{2}$ or acetate during net methane production when grown with methanogenic substrates in the absence of exogenous electron acceptors ${ }^{13,14}$. Although TMO is likely dependent on reversal of carbon transformation reactions in acetoclastic or $\mathrm{CO}_{2}$-reducing methanogenic pathways (Fig. 5), endergonic reactions of TMO are driven by energy conservation dependent on methanogenesis rather than reduction of $\mathrm{Fe}(\mathrm{III})$.

\section{Methods}

Cell growth and materials. Methanosarcina acetivorans wild-type strain C2A, isolated from marine sediment, was grown with acetate or methanol as previously described $^{55,56}$. The CO dehydrogenase/acetyl-CoA synthase (CODH/ACS) was purified from acetate-grown cells as previously described ${ }^{43}$. The ferredoxin (Fdx) upregulated in acetate-grown $M$. acetivorans was heterologously produced and purified as previously described ${ }^{43}$. Purification of $\mathrm{F}_{420}$ from methanol-grown cells and preparation of $\mathrm{F}_{420} \mathrm{H}_{2}$ was as described elsewhere ${ }^{57}$. AQDS was purchased from ACROS Organics and HSCoB from Tocris Bioscience. CoMS-SCoB heterodisulfide was prepared as described elsewhere ${ }^{57}$. HSCoM, THF and $\mathrm{CH}_{3}$-THF were purchased from Sigma Chemical.

Membrane fragments were isolated as described previously ${ }^{27}$. Washed everted membrane vesicles were prepared as described elsewhere except $5 \mathrm{mM}$ ferric citrate or $5 \mathrm{mM}$ AQDS were included in buffers prior to French pressure cell lysis to load vesicles with either or both electron acceptor where indicated ${ }^{25,57}$. The integrity of vesicles was determined by artificial energization as described elsewhere ${ }^{30}$.
Maximum quenching of acridine orange was within $5 \mathrm{~s}$ followed by $37 \%$ dequenching over a period of $4 \mathrm{~min}$.

Activity assays. All activity assays were performed anaerobically at $21^{\circ} \mathrm{C}$ in serum-stoppered glass vials with the indicated gas atmosphere. Pre-reduced $\mathrm{Fdx}$ was prepared by incubation with CODH/ACS in $1.0 \mathrm{Atm}$ CO followed by replacement of $\mathrm{CO}$ with Ar. The same procedure was used for assays requiring continuous reduction of $\mathrm{Fdx}$ except the $\mathrm{CO}$ was present throughout the assay. Additions and samplings were performed with gas-tight syringes. Contents of reaction mixtures are included in Figure captions.

The reduced ferredoxin $\left(\mathrm{Fdx}_{\mathrm{R}}\right)$ : $\mathrm{Fe}(\mathrm{III})$ oxidoreductase activity of vesicles was monitored spectrophotometrically following the oxidation of pre-reduced Fdx. Activity was based on the change of absorbance at $410 \mathrm{~nm}\left(\varepsilon=30.0 \mathrm{mM}^{-1} \mathrm{~cm}^{-1}\right)$. Measurement of $\mathrm{Na}^{+}$translocation was as previously described with the following modifications ${ }^{25}$. The reaction mixture was incubated in $1.0 \mathrm{Atm}$ CO for $40 \mathrm{~min}$ to ensure $\mathrm{Na}^{+}$equilibrium prior to addition of CODH/ACS to start the reaction. At the time points indicated, the external ${ }^{22} \mathrm{Na}$ was removed from samples $(100 \mu \mathrm{l})$ using a column $(0.5 \times 3 \mathrm{~cm})$ of DOWEX 50WX8 (Sigma). The vesicles were eluted with $1.0 \mathrm{ml}$ of $0.4 \mathrm{M}$ sucrose followed by addition of $9.0 \mathrm{ml}$ of Ultima Gold LLT scintillation fluid (Perkin Elmer) and measurement of radioactivity with a LSC Beckman Coulter Model LS6500 liquid scintillation counter. The $\mathrm{Na}^{+}$ concentration of the reaction mixture was determined with a Ross Sure-Flow (Thermo Fisher Scientific) $\mathrm{Na}^{+}$-selective combination electrode.

$\mathrm{F}_{420} \mathrm{H}_{2}: \mathrm{Fe}$ (III) oxidoreductase activity of vesicles was monitored spectrophotometrically following oxidation of $\mathrm{F}_{420} \mathrm{H}_{2}$. Activity was based on the change of absorbance at $420 \mathrm{~nm}\left(\varepsilon=41.4 \mathrm{mM}^{-1} \mathrm{~cm}^{-1}\right)$. Measurement of $\mathrm{H}^{+}$ translocation was as described elsewhere ${ }^{57}$ with the following modifications. An Inlab Micro $^{\mathrm{TM}}$ (Mettler-Toledo) $\mathrm{pH}$ electrode was connected to a EPU353 $\mathrm{pH} / \mathrm{ISE}$ isoPOD ${ }^{\mathrm{TM}}$ (eDAQ) $\mathrm{pH}$ meter interfaced to a computer through Pod-Vu ${ }^{\mathrm{TM}}$ software (eDAQ) to monitor the $\mathrm{pH}$ change with time. Additions of $\mathrm{F}_{420} \mathrm{H}_{2}$ were made with a gas-tight syringe through the rubber stopper. The $\mathrm{pH}$ changes were calibrated with standard solutions of $\mathrm{KOH}$.

The forward and reverse $\mathrm{CH}_{3}$-THF:HSCoM methyltransferase activities of vesicles were performed as described elsewhere ${ }^{29}$. Forward activity was monitored by detection of thiol group consumption on transfer of the methyl group from $\mathrm{CH}_{3}$-THF to HSCoM. Reverse activity was monitored by detection of HSCoM production on transfer of the methyl group of $\mathrm{CH}_{3}-\mathrm{SCoM}$ to THF.

Reverse heterodisulfide reductase activity (HSCoM $+\mathrm{HSCoB} \rightarrow \mathrm{CoMS}-\mathrm{SCoB}+2 \mathrm{H}$ ${ }^{+}$) was assayed as previously described except activity was monitored by consumption of thiol groups ${ }^{58}$.

Analytical. Production or consumption of HSCoM and HSCoB was determined with Ellman's reagent ${ }^{59}$. Determination of Fe(II) was by the ferrozine method described elsewhere ${ }^{60}$. Vesicles were collected from the reaction mixture by centrifuging at $135,000 \times g$ for $60 \mathrm{~min}$. CHAPS $(15 \mathrm{mM})$ was added to dissolve vesicles and release $\mathrm{Fe}(\mathrm{II})$ prior to analysis. Protein was estimated with the Bradford assay kit (Bio-Rad Laboratories) using bovine serum albumin as the protein standard ${ }^{61}$.

Data availability. All relevant data are available from the authors.

Received: 4 July 2017 Accepted: 4 April 2018

Published online: 24 April 2018

\section{References}

1. Conrad, R. The global methane cycle: recent advances in understanding the microbial processes involved. Environ. Microbiol. Rep. 1, 285-292 (2009).

2. Reeburgh, W. S. Oceanic methane biogeochemistry. Chem. Rev. 107, 486-513 (2007).

3. Rhee, T. S., Kettle, A. J. \& Andreae, M. O. Methane and nitrous oxide emissions from the ocean: a reassessment using basin-wide observations in the Atlantic. J. Geophys. Res. 114 https://doi.org/10.1029/2008JD011662 (2009).

4. Valentine, D. L. Biogeochemistry and microbial ecology of methane oxidation in anoxic environments: a review. Antonie Van Leeuwenhoek 81, 271-282 (2002).

5. McGlynn, S. E. Energy metabolism during anaerobic methane oxidation in ANME archaea. Microbes Environ. 32, 5-13 (2017).

6. Timmers, P. H. et al. Reverse methanogenesis and respiration in methanotrophic Archaea. Archaea 2017, 1654237 (2017).

7. Scheller, S., Yu, H., Chadwick, G. L., McGlynn, S. E. \& Orphan, V. J. Artificial electron acceptors decouple archaeal methane oxidation from sulfate reduction. Science 351, 703-707 (2016).

8. Rotaru, A. E. \& Thamdrup, B. A new diet for methane oxidizers. Science 351, 658 (2016). 
9. Knittel, K. \& Boetius, A. Anaerobic oxidation of methane: progress with an unknown process. Annu. Rev. Microbiol. 63, 311-334 (2009).

10. Ettwig, K. F. et al. Archaea catalyze iron-dependent anaerobic oxidation of methane. Proc. Natl. Acad. Sci. USA 113, 12792-12796 (2016).

11. Beal, E. J., House, C. H. \& Orphan, V. J. Manganese- and iron-dependent marine methane oxidation. Science 325, 184-187 (2009).

12. Haroon, M. F. et al. Anaerobic oxidation of methane coupled to nitrate reduction in a novel archaeal lineage. Nature 500, 567-570 (2013).

13. Moran, J. J., House, C. J., Thomas, B. \& Freeman, K. H. Products of trace methane oxidation during nonmethyltrophic growth by Methanosarcina. J. Geophys. Res. 112, https://doi.org/10.1029/2006JG000268 (2007).

14. Moran, J. J., House, C. H., Freeman, K. H. \& Ferry, J. G. Trace methane oxidation studied in several Euryarchaeota under diverse conditions. Archaea 1, 303-309 (2005).

15. Soo, V. W. et al. Reversing methanogenesis to capture methane for liquid biofuel precursors. Microb. Cell. Fact. 15, 11 (2016).

16. Clomburg, J. M., Crumbley, A. M. \& Gonzalez, R. Industrial biomanufacturing: the future of chemical production. Science 355, aag0804 (2017).

17. McAnulty, M. J. et al. Metabolic engineering of Methanosarcina acetivorans for lactate production from methane. Biotechnol. Bioeng. 114, 852-861 (2016).

18. McAnulty, M. J. et al. Electricity from methane by reversing methanogenesis. Nat. Commun. 8, 15419 (2017)

19. McGlynn, S. E., Chadwick, G. L., Kempes, C. P. \& Orphan, V. J. Single cell activity reveals direct electron transfer in methanotrophic consortia. Nature 526, 531-535 (2015).

20. Arshad, A. et al. A metagenomics-based metabolic model of nitrate-dependent anaerobic oxidation of methane by Methanoperedens-like Archaea. Front. Microbiol. 6, 1423 (2015).

21. Wang, F. P. et al. Methanotrophic archaea possessing diverging methaneoxidizing and electron-transporting pathways. ISME J. 8, 1069-1078 (2014).

22. Ferry, J. G. How to make a living exhaling methane. Annu. Rev. Microbiol. 64 453-473 (2010).

23. Welte, C. \& Deppenmeier, U. Bioenergetics and anaerobic respiratory chains of aceticlastic methanogens. Biochim. Biophys. Acta 1837, 1130-1147 (2014).

24. Buan, N. R. \& Metcalf, W. W. Methanogenesis by Methanosarcina acetivorans involves two structurally and functionally distinct classes of heterodisulfide reductase. Mol. Microbiol. 75, 843-853 (2010).

25. Schlegel, K., Welte, C., Deppenmeier, U. \& Muller, V. Electron transport during aceticlastic methanogenesis by Methanosarcina acetivorans involves a sodium-translocating Rnf complex. FEBS J. 279, 4444-4452 (2012).

26. $\mathrm{Li}, \mathrm{Q}$. et al. Electron transport in the pathway of acetate conversion to methane in the marine archaeon Methanosarcina acetivorans. J. Bacteriol. 188, 702-710 (2006).

27. Wang, M., Tomb, J. F. \& Ferry, J. G. Electron transport in acetate-grown Methanosarcina acetivorans. BMC Microbiol. 11, 165 (2011).

28. Li, L. et al. Quantitative proteomic and microarray analysis of the archaeon Methanosarcina acetivorans grown with acetate versus methanol. J. Proteome Res. 6, 759-771 (2007).

29. Becher, B., Muller, V. \& Gottschalk, G. $N^{5}$ methyl-tetrahydromethanopterin: coenzyme M methyltransferase of Methanosarcina strain $\mathrm{Gol}$ is an $\mathrm{Na}^{+}$. translocating membrane protein. J. Bacteriol. 174, 7656-7660 (1992).

30. Schlegel, K., Leone, V., Faraldo-Gomez, J. D. \& Muller, V. Promiscuous archaeal ATP synthase concurrently coupled to $\mathrm{Na}^{+}$and $\mathrm{H}^{+}$translocation. Proc. Natl. Acad. Sci. USA 109, 947-952 (2012).

31. Deppenmeier, U., Blaut, M., Mahlmann, A. \& Gottschalk, G. Reduced coenzyme $\mathrm{F}_{420}$ :heterodisulfide oxidoreductase, a proton-translocating redox system in methanogenic bacteria. Proc. Natl. Acad. Sci. USA 87, 9449-9453 (1990).

32. Hallam, S. J. et al. Reverse methanogenesis: testing the hypothesis with environmental genomics. Science 305, 1457-1462 (2004).

33. Lessner, D. J. et al. An unconventional pathway for reduction of $\mathrm{CO}_{2}$ to methane in CO-grown Methanosarcina acetivorans revealed by proteomics. Proc. Natl. Acad. Sci. USA 103, 17921-17926 (2006).

34. Greening, C. et al. Physiology, biochemistry, and applications of $\mathrm{F}_{420^{-}}$and $\mathrm{F}_{\mathrm{O}^{-}}$ dependent redox reactions. Microbiol. Mol. Biol. Rev. 80, 451-493 (2016).

35. Deppenmeier, U. The membrane-bound electron transport system of Methanosarcina species. J. Bioenerg. Biomembr. 36, 55-64 (2004).

36. Ferry, J. G. in Bioenergy (eds J. D. Wall et al.) 155-170 (ASM Press, Washington, 2008)

37. Li, Q., Li, L., Rejtar, T., Karger, B. L. \& Ferry, J. G. The proteome of Methanosarcina acetivorans. Part II, comparison of protein levels in acetateand methanol-grown cells. J. Proteome Res. 4, 129-136 (2005).

38. Arbing, M. A. et al. Structure of the surface layer of the methanogenic archaean Methanosarcina acetivorans. Proc. Natl. Acad. Sci. USA 109, 11812-11817 (2012).
39. Shi, L. et al. Molecular underpinnings of Fe(III) oxide reduction by Shewanella Oneidensis MR-1. Front. Microbiol. 3, 50 (2012).

40. White, G. F. et al. Rapid electron exchange between surface-exposed bacterial cytochromes and Fe(III) minerals. Proc. Natl. Acad. Sci. USA 110, 6346-6351 (2013).

41. Lovley, D. R., Coates, J. D., Blunt-Harris, E. L., Phillips, E. J. P. \& Woodward, J. C. Humic substances as electron acceptors for microbial respiration. Nature 382, 445-448 (1996).

42. Lovley, D. R. et al. Humic substances as a mediator for microbially catalyzed metal reduction. Acta Hydrochim. Hydrobiol. 26, 152-157 (1998).

43. Yan, Z., Wang, M. \& Ferry, J. G. A Ferredoxin- and $\mathrm{F}_{420} \mathrm{H}_{2}$-dependent, electron-bifurcating, heterodisulfide reductase with homologs in the domains Bacteria and Archaea. mBio 8, e02285-16 (2017).

44. Jasso-Chavez, R., Apolinario, E. E., Sowers, K. R. \& Ferry, J. G. MrpA functions in energy conversion during acetate-dependent growth of Methanosarcina acetivorans. J. Bacteriol. 195, 3987-3994 (2013).

45. Nayak, D. D. \& Metcalf, W. W. Cas9-mediated genome editing in the methanogenic archaeon Methanosarcina acetivorans. Proc. Natl. Acad. Sci. USA 114, 2976-2981 (2017).

46. Mueller, T. J. et al. Methane oxidation by anaerobic archaea for conversion to liquid fuels. J. Ind. Microbiol. Biotechnol. 42, 391-401 (2014).

47. Nazem-Bokaee, H., Gopalakrishnan, S., Ferry, J. G., Wood, T. K. \& Maranas, C. D. Assessing methanotrophy and carbon fixation for biofuel production by Methanosarcina acetivorans. Microb. Cell. Fact. 15, 10 (2016).

48. Heuer, V. et al. Online delta C-13 analysis of volatile fatty acids in sediment/ porewater systems by liquid chromatography-isotope ratio mass spectrometry. Limnol. Oceanogr. Meth. 4, 346-357 (2006).

49. Weber, H. S., Habicht, K. S. \& Thamdrup, B. Anaerobic methanotrophic Archaea of the ANME-2d cluster are active in a low-sulfate, iron-rich freshwater sediment. Front. Microbiol. 8, 619 (2017).

50. Scheller, S., Goenrich, M., Boecher, R., Thauer, R. K. \& Jaun, B. The key nickel enzyme of methanogenesis catalyses the anaerobic oxidation of methane. Nature 465, 606-608 (2010).

51. Marsili, E. et al. Shewanella secretes flavins that mediate extracellular electron transfer. Proc. Natl. Acad. Sci. USA 105, 3968-3973 (2008).

52. Berger, S., Frank J., Dalcin Martins P., Jetten, M. S. M. \& Welte, C. Highquality draft genome sequence of "Candidatus Methanoperedens sp." strain BLZ2, a nitrate-reducing anaerobic methane-oxidizing archaeon enriched in an anoxic bioreactor. Genome Announc. 5, e01159-17 (2017).

53. Wegener, G., Krukenberg, V., Riedel, D., Tegetmeyer, H. E. \& Boetius, A. Intercellular wiring enables electron transfer between methanotrophic archaea and bacteria. Nature 526, 587-590 (2015).

54. Berg, I. A. et al. Autotrophic carbon fixation in archaea. Nat. Rev. Microbiol. 8, 447-460 (2010).

55. Sowers, K. R., Baron, S. F. \& Ferry, J. G. Methanosarcina acetivorans sp. nov., an acetotrophic methane-producing bacterium isolated from marine sediments. Appl. Environ. Microbiol. 47, 971-978 (1984).

56. Sowers, K. R., Nelson, M. J. K. \& Ferry, J. G. Growth of acetotrophic, methaneproducing bacteria in a $\mathrm{pH}$ auxostat. Curr. Microbiol. 11, 227-230 (1984).

57. Welte, C. \& Deppenmeier, U. Proton translocation in methanogens. Methods Enzymol. 494, 257-280 (2011).

58. Peer, C. W., Painter, M. H., Rasche, M. E. \& Ferry, J. G. Characterization of a CO:heterodisulfide oxidoreductase system from acetate-grown Methanosarcina thermophila. J. Bacteriol. 176, 6974-6979 (1994).

59. Ellman, G. L. A colorimetric method for determining low concentrations of mercaptans. Arch. Biochem. Biophys. 74, 443-450 (1958).

60. Yamada, C., Kato, S., Kimura, S., Ishii, M. \& Igarashi, Y. Reduction of Fe(III) oxides by phylogenetically and physiologically diverse thermophilic methanogens. FEMS Microbiol. Ecol. 89, 637-645 (2014).

61. Bradford, M. M. A rapid and sensitive method for the quantitation of microgram quantities of protein utilizing the principle of protein-dye binding. Anal. Biochem. 72, 248-254 (1976).

\section{Acknowledgements}

This work was supported by: the Department of Energy Advanced Research Projects Agency-Energy \#0881-1525 to J.G.F. (70\%); the Division of Chemical Sciences, Geosciences and Biosciences, Office of Basic Energy Sciences of the US Department of Energy through grant DE-FG02-95ER20198 (20\%); the Person endowment to J.G.F. (10\%); and the US National Science Foundation Division of Earth Sciences (award GEO-1451593) to C.A.G.

\section{Author contributions}

J.G.F. and Z.Y. devised the study. Z.Y. conducted the experiments. P.J. and C.A.G. assisted with the ${ }^{22} \mathrm{Na}$ experiments. J.G.F. and Z.Y. analyzed the data and wrote the manuscript. 


\section{Additional information}

Supplementary Information accompanies this paper at https://doi.org/10.1038/s41467018-04097-9.

Competing interests: The authors declare no competing interests.

Reprints and permission information is available online at http://npg.nature.com/ reprintsandpermissions/

Publisher's note: Springer Nature remains neutral with regard to jurisdictional claims in published maps and institutional affiliations. (c) (i) Open Access This article is licensed under a Creative Commons Attribution 4.0 International License, which permits use, sharing, adaptation, distribution and reproduction in any medium or format, as long as you give appropriate credit to the original author(s) and the source, provide a link to the Creative Commons license, and indicate if changes were made. The images or other third party material in this article are included in the article's Creative Commons license, unless indicated otherwise in a credit line to the material. If material is not included in the article's Creative Commons license and your intended use is not permitted by statutory regulation or exceeds the permitted use, you will need to obtain permission directly from the copyright holder. To view a copy of this license, visit http://creativecommons.org/ licenses/by/4.0/.

(C) The Author(s) 2018 